\title{
Curcumin Protects against Monosodium Glutamate Neurotoxicity and Decreasing NMDA2B and mGluR5 Expression in Rat Hippocampus
}

\author{
Rania M. Khalila Naglaa F. Khedr ${ }^{b}$ \\ aBiochemistry Department, Faculty of Pharmacy, AL-Delta University, Gamasa, ${ }^{b B i o c h e m i s t r y ~}$ \\ Department, Faculty of Pharmacy, Tanta University, Tanta, Egypt
}

\section{Key Words}

Brain toxicity $\cdot$ Cholinesterase $\cdot$ Curcumin $\cdot$ Monosodium glutamate $\cdot$ mGLUTR5 $•$ NMDA2B

\begin{abstract}
Background: Monosodium glutamate (MSG) is a flavor enhancer used in food industries. MSG is well documented to induce neurotoxicity. Curcumin (CUR) reportedly possesses beneficial effects against various neurotoxic insults. Hence, this present study has been designed to evaluate the neuroprotective effect of curcumin on MSG-induced neurotoxicity in rats. Methods: Thirty-two male Wister rats were divided into four groups $(n=8)$ : Control group, MSG group, CUR group and MSG + CUR group. CUR (Curcumin $150 \mathrm{mg} / \mathrm{kg}$, orally) was given day after day for four weeks along with MSG (4 mg/kg, orally). After 4 weeks, rats were sacrificed and brain hippocampus was isolated immediately on ice. Inflammatory marker TNF $\alpha$ and acetylcholinesterase (AChE) activity (marker for cholinergic function) were estimated. Gene expressions of metabotropic glutamate receptor 5 (mGluR5) and N-methyl-D-aspartate receptor 2B (NMDA2B) along with glutamate concentration were assessed. Results: Treatment with CUR significantly attenuated AChE activity and TNF $\alpha$ in MSG-treated animals. The antiinflammatory properties of CUR may be responsible for this observed neuroprotective action. A possible role of CUR to attenuate both glutamate level and gene expression of NMDA2B and mGLUR5 in brain hippocampus was established when compared to MSG group. Conclusion: We concluded that CUR as flavor enhancer protects against MSG-induced neurotoxicity in rats.

\section{Introduction}

Monosodium Glutamate (MSG) is one of the world's most used flavor enhancer (food additive number E621). It increases the sapidity of food. It elicits a taste described in Japanese as umami, which is translated to "savory" [1]. The average daily intake of MSG per person in industrialized countries is $0.3-1.0 \mathrm{~g}$ but in many countries there are no limitations 
on the amount of it which is questioned due to its toxic effects. MSG is demonstrated as the Chinese restaurant syndrome that causes symptoms such as numbness, weakness, flushing, sweating, dizziness, and headache [2].

Glutamate is abundant excitatory neurotransmitter in the brain and plays an important role in both physiological and pathological processes. Glutamate may act as a potent neurotoxin in excessive amounts through increasing the excitability and by activating the proteolytic enzymes [3]. Glutamate acts through ionotropic and metabotropic glutamate receptors. There are three families of ionotropic receptors: N-methyl-D-aspartate (NMDA), $\alpha$-amino-3- hydroxy-5-methyl-4-isoxazolepropionic acid (AMPA) and kainite receptors and three groups of metabotropic glutamate receptors (mGluRs) [4]. mGluRs participate in synaptic transmission and synaptic plasticity in the hippocampus [5]. mGluRs belong to the G-protein-coupled receptor (GPCR) superfamily and are sub-classified into three groups with eight subtypes based on sequence homology, G-protein coupling, and ligand selectivity. Group I mGluRs include mGluR1 and mGluR5, both of which are abundant in the adult hippocampus and cerebral cortex, which are critical regions in the brain for learning and memory processes [6].

Otherwise, beside the vital role of hippocampus in memory formation, and regulation of behavior; it is involved in increasing levels of pro-inflammatory cytokines as TNF- $\alpha$, which contributes to the brain damage and death of neurons [7]. Under physiological conditions, cytokines play an important role in neurogenesis, synaptic plasticity, long-term potentiation and memory formation and consolidation. On the other hand, the over-expression of these cytokines in the CNS is associated with behavioral and cognitive impairment in several pathological conditions. Anti-inflammatory management seems effective on cognitive deficit [8]. However, the molecular mechanisms of MSG-induced learning and memory impairments can alter the levels of some neurotransmitters and activity of neurotransmitter metabolism related enzymes, such as acetyl cholinesterase (AChE) and dopamine $\beta$-hydroxylase, in the brain [9]. AChE is associated with brain development, learning, memory and neuronal damage. AChE activation leads to a fast acetylcholine degradation and a subsequent down stimulation of ACh receptors causing undesirable effects on cognitive functions [10].

Complementary medicine is widely used to provide favorable effects including reduction of toxicity, improvement of diseases-related symptoms and enhancing the immune system [11]. The problems associated with MSG intake make a desire to combine it with other compound to reduce its toxicity without affecting its flavor acceptability. Curcumin, the principal polyphenol of the spice turmeric (Curcuma longa), exhibits anti-inflammatory, antioxidant, antitumor, neuroprotective and antiamyloidogenic properties [12]. Because of the potential ability of curcumin to preserve cognition in the elderly, we aimed to assess the effect of curcumin on MSG neurotoxicity elicited by activation of mGLUR and NMDAs genes in rat hippocampus.

\section{Materials and Methods}

Monosodium glutamate (MSG) powder of purity $\geq 99 \%$ was purchased from Ajinomoto Co. Inc. (Japan). Curcumin powder ( $\geq 94 \%$ curcuminoid content, $\geq 80 \%$ curcumin) was purchased from Sigma Aldrich ((ID. C7727, MO.USA). All other chemicals used were of high analytical grade.

Animal treatment and experimental design

The study was performed in accordance with the guidelines for the care and use of laboratory animals approved by Research Ethical Committee (Faculty of pharmacy, Tanta University, Egypt). Thirty-two male Wistar rats weighing 120-150 g (12 week-old) were obtained from National Research Center (Cairo, Egypt) and maintained with a 12-h light/dark cycle at controlled temperature $\left(22 \pm 2{ }^{\circ} \mathrm{C}\right)$ and allowed free access to standard rat chow and tap water. Rats were divided into 4 groups according to treatment as the following:

Control $(n=8)$ : Rats were received vehicle $(0.7 \%$ carboxymethylcellulose; $C M C)$ via orogastric gavage once day after day for four successive weeks. 
Khalil/Khedr: Modulation of Monosodium Glutamate Neurotoxicity by Curcumin

CUR group (n=8): Each rat was received $150 \mathrm{mg} / \mathrm{kg}$ curcumin suspended in $0.7 \%$ CMC via orogastric gavage once day after day for four successive weeks.

MSG group (n=8): rats were received $4 \mathrm{mg} / \mathrm{kg}$ MSG dissolved in distilled water via orogastric gavage once day after day for four successive weeks.

MSG + CUR (n=8): rats were received same doses of MSG and CUR via orogastric gavage once day after day for four successive weeks.

At the end of the experimental period, rats were sacrificed via decapitation under light ether anesthesia. The entire hippocampus was removed and homogenized in ice-cold homogenizing buffer (1:4 w/v) (137 mM NaCl, 20 mM Tris-HCl (pH 8.0), 1\% NP40, 10\% glycerol, 1 mM PMSF, 10 mg/mL aprotinin, $1 \mu \mathrm{g} / \mathrm{mL}$ leupetin, and $0.5 \mathrm{mM}$ sodium vanadate). The homogenates of hippocampus were centrifuged at $14,000 \times \mathrm{g}$ for $25 \mathrm{~min}$ at $4^{\circ} \mathrm{C}$ [13]. Then, supernatants were separated and used for assay of TNF- $\alpha$ and acetylcholinesterase activity. The hippocampus of the other hemisphere was stored at $-80^{\circ} \mathrm{C}$ and used for RT-PCR experiments and glutamate determination.

Determination of Acetylcholinesterase (AChE; EC 3.1.1.7.) activity in hippocampus

Acetylcholinesterase (AChE) activity was assessed using the method of Ellman et al. [14]. $250 \mu \mathrm{L}$ of

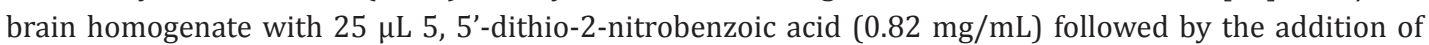
$25 \mu \mathrm{L}$ of the enzyme substrate; acetylthiocholine $(6.7 \mathrm{mg} / \mathrm{mL})$. The change in absorbance/min during a 5 min interval was measured at $405 \mathrm{~nm}$. Enzyme activity was calculated using the estimated extinction coefficient $\varepsilon=14,150 \mathrm{M}^{-1} \mathrm{~cm}^{-1}$. Enzyme activity was expressed in $\mu$ moles of acetylthiocholine hydrolyzed/ $\mathrm{min} / \mathrm{mg}$ tissue.

Determination of tumor necrosis factor- $\alpha$ (TNF- $\alpha)$ in hippocampus

Level of TNF- $\alpha$ in brain homogenate was determined using ELISA kit (Glory science CO., LTD, China) by a microplate reader (Tecan, Infinite 200 PRO, Switzerland). TNF- $\alpha$ level (ng/L) was calculated according to standard curve as described by the manufacture. The detection limit of assays was $4 \mathrm{ng} / \mathrm{L}$ for TNF- $\alpha$.

\section{Determination glutamate neurotransmitter Level in hippocampus}

Brain hippocampal tissue was homogenized in PBS (10mg to $100 \mu \mathrm{L}$ PBS). After that, homogenates were centrifuged for $15 \mathrm{~min}$ at $1500 \times \mathrm{g}$. Supernatants were collected immediately for glutamate measurement using rat glutamate ELISA kit (No: MBS047402, MyBioSource, Canada) by a microplate reader (Tecan, Infinite 200 PRO, Switzerland) at $450 \mathrm{~nm}$. Glutamate levels were calculated using standard curve as described by the manufacturer. Limit of detection was $2.5 \mu \mathrm{mol} / \mathrm{L}$ up to $700 \mu \mathrm{mol} / \mathrm{L}$.

Gene expression of mGLUT5 and NMDA2B in hippocampus by quantitative real-time PCR ( $q R T$-PCR)

Total RNA was extracted from brain hippocampus using TRIZOL® reagent $\left(\right.$ Ambion $^{\mathrm{TM}}$, life technologies, Canda). RNA concentration was determined by measuring absorbance at $260 \mathrm{~nm}$ using Nanodrop 2000 spectrophotometer (Thermo Fisher Scientific Inc., USA). C DNA was prepared from $0.5 \mu \mathrm{g}$ total RNA by reverse transcription using TIANScript RT Kit (Tiangen ${ }^{\circ}$ Biotech, CO., LTD, China). The primers used for amplification of mGLUT5, NMDA2B and $\beta$-actin primers were as follows [15]: mGLUT5 forward, 5`-CACTCTTGCCCAACATCAC-3`and reverse, 5`-CACAGCGTACCAAACCTTC-3`; NMDA2B forward, 5'-GGATCTACCAGTCTAACATG-3`, 3`-GTCACCCTAGTGATTGATAG-5`; $\beta$-actin forward, $5 `$-AGCCATGTACGTAGCCATCC-3` and reverse, $5^{`}$-ACCCTCATAGATGGGCACAG-3`. Real-time PCR was done in an ICycler thermocycler (BioRad, Mississauga, ON, Canada) with SYBR Premix Ex Taq (Takara Bio, Shiga, Japan). The expression levels of the mGLUT5 and NMDA2B genes were normalized to the $\beta$-actin gene expression level.

\section{Statistical Analysis}

Data are represented as mean \pm SD. Statistical analysis was performed using the SPSS v.16.0 software for Windows [16]. Groups variance were analyzed by one-way Analysis of Variation (ANOVA) and Fisher least significant difference test (LSD) was tested for significance. Differences were considered as significant at $\mathrm{P}<0.05$. 


\section{Results}

Effect of CUR on hippocampus concentration of glutamate in MSG treated rats

Figure 1 shows that the tissue level of glutamate was significantly increased during the MSG administration $(525 \pm 41.6 \mu \mathrm{mol} / \mathrm{L}, \mathrm{P}<0.01)$ when compared to control group. Otherwise, the level of glutamate was significantly decreased in presence of CUR supplementation in spite that it not returned to normal $(465.1 \pm 19.9 \mu \mathrm{mol} / \mathrm{L}, \mathrm{P}<0.01)$.

\section{Effect of CUR on hippocampus AChE activity in treated groups}

Figure 2 illustrates that MSG group showed a significant elevation in AChE level $(183.6 \pm 33.5 \mu \mathrm{mol} / \mathrm{min} / \mathrm{mg}$ tissue, $\mathrm{P}<0.05)$ compared to control group. However, both CUR and MSG+CUR groups showed a significant decrease in AChE activity (137.4 \pm 28.67 , 134.28 $\pm 16.7, \mathrm{P}<0.01$ ), respectively compared to MSG group.

\section{Effect of CUR on hippocampus TNF $\alpha$ level in treated groups}

Figure 3 shows that while MSG group had significantly elevated TNFa level (52.1 $\pm 10.7 \mathrm{ng} / \mathrm{L}, \mathrm{P}<0.001)$ compared to control group; both CUR \& MSG+ CUR groups showed a significant lower TNF $\alpha$ level $(35 \pm 9.1,39.3 \pm 9.7 \mathrm{ng} / \mathrm{L}, \mathrm{P}<0.01$, respectively) compared to MSG group, otherwise, MSG+CUR group represents a significant elevation in TNF $\alpha$ level when compared to control group.

Effect of CUR on hippocampus NMDA2B and mGLUR5 gene expression in treated groups

Figure $4 \& 5$ represents that while MSG group was showing a significant elevation in NMDA2B expression $(6.2 \pm 0.1, \mathrm{P}<0.001)$ compared to control group, both CUR $(1.7 \pm 0.8$,

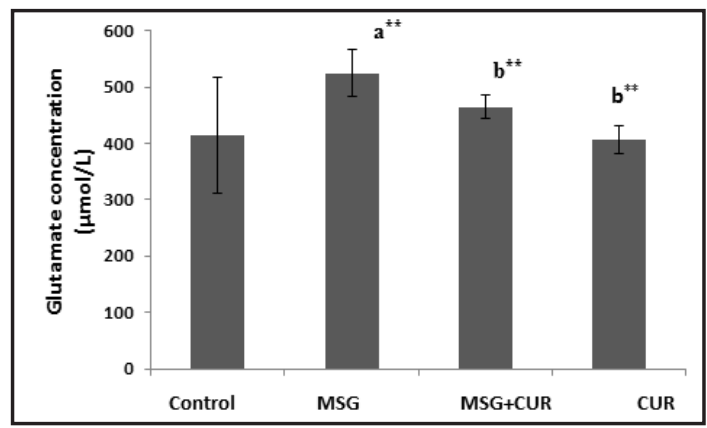

Fig. 1. Levels of glutamate in brain hippocampus of treated groups. ${ }^{* *}: \mathrm{P}<0.01$, a: significant versus control group, b: significant versus MSG group, MSG: monosodium glutamate, CUR: Curcumin.

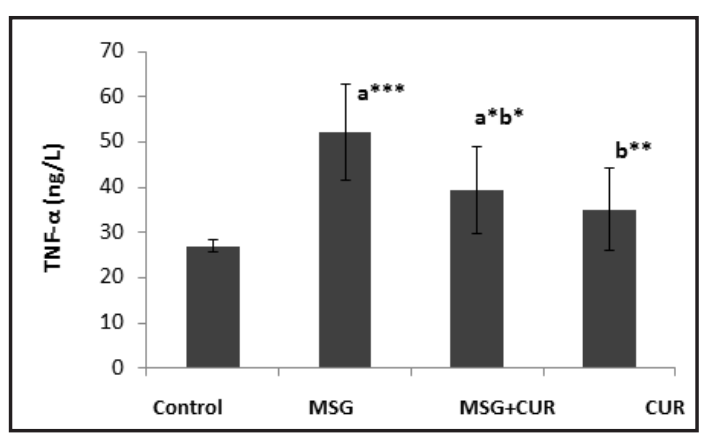

Fig. 3. Levels of TNF $\alpha$ in brain hippocampus of treated groups. a: significant versus control group, b: significant versus MSG group, *: $\mathrm{p}<0.05,{ }^{* *}$ : $\mathrm{P}<0.01,{ }^{* * *}$ : $\mathrm{P}<$ 0.001, MSG: monosodium glutamate, CUR: Curcumin.

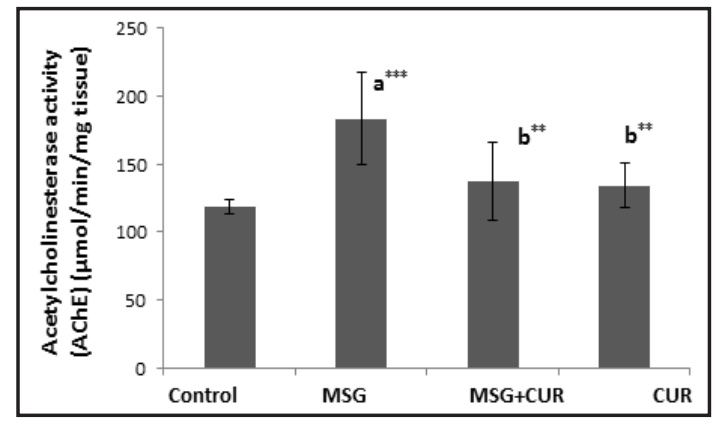

Fig. 2. Acetylcholinesterase (AChE) activity in brain hippocampus of treated groups. a: significant versus control group, b: significant versus MSG group, ${ }^{* *}$ : $\mathrm{P}<0.01$, ***: $\mathrm{P}<$ 0.001, MSG: monosodium glutamate, CUR: Curcumin.

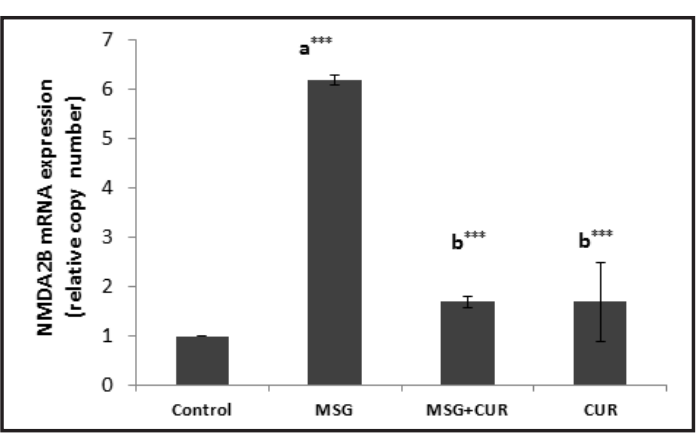

Fig. 4. NMDA2B gene expression in brain hippocampus of treated groups. ${ }^{* * *} \mathrm{P}<0.001$, a: significant versus control group, b: significant versus MSG group. MSG: monosodium glutamate, CUR: Curcumin. 
$\mathrm{P}<0.001)$ \& MSG+ CUR $(1.7 \pm 0.11, \mathrm{P}<0.001)$ groups showed a significant decrease in NMDA2B expression compared to MSG group. Otherwise, CUR significantly decreased mGLUR5 gene expression either alone $(0.6 \pm 0.025, \mathrm{P}<0.009)$ or in combination with MSG $(0.9 \pm 0.03, \mathrm{P}<0.05)$ compared to MSG group alone.

\section{Discussion}

Geha etal. [17] reported that MSG a flavor enhancer produces neuronal symptoms such as numbness, weakness, dizziness and headaches. Earlier studies reported that MSG alters mitochondrial lipid peroxidation and antioxidant status in cerebral hemispheres, cerebellum, brain stem and diencephalon and causes damage to hypothalamic neurons in rat brains $[18,19]$.

Glutamate elicits synaptic responses via metabotropic mGLU and ionotropic NMDA receptors leading to increased cellular excitation and induce the release of $\mathrm{Ca}^{+2}$ from intracellular stores. On the other hand, activation of ionotropic receptors increases cellular permeability to $\mathrm{Ca}^{+2}$ [3]. In the present study, glutamate concentration was remarkably increased in MSG treated rats. The amino acid transporters present in neurons and glial cells are very important for the normal function of glutamatergic transmission and for the maintenance of extracellular glutamate levels below potentially excitotoxic concentrations [20]. The possible reasons for increased glutamate level in MSG treated rats is due to blockade of re-uptake of glutamate by glutamate transporter in the presynaptic neurons and decreased downstream enzymes such as glutamate decarboxylase for the inhibitory neurotransmitter gamma amino butyric acid synthesis [21].

It was reported that there is association between increased glutamate neurotransmission and cognitive dysfunction. Previous studies report that MSG causes severe behavioral abnormalities such as increased irritability, hypoactivity, and deficits in spontaneous alternation behavior [22]. In agreement with the above reports, CUR may attenuate the extracellular glutamate concentration at safe physiological level which may be by a number of buffering mechanisms include uptake of glutamate by glial cells and its conversion by glutamine synthetase or glutamate decarboxylase to the nontoxic glutamine [23].

However, glutamate excitotoxicity involves over-activation of NMDA2B and mGLUR5 receptors may lead to release of $\mathrm{Ca}^{+2}$ from the intracellular stores, which causes over activation of enzymes like protein kinases leading to the degradation of proteins and membranes, thus enhancing cellular damage [24]. Our results showed a marked decrease of both mGluR5 and NMDA2B receptor expression in rat hippocampus in MSG combined with CUR and CUR alone-exposed rats. The observed CUR-mediated inhibition of glutamate release results in down-regulation receptors expression of both mGluR5 and NMDA2B $[25,26]$.

Acetyl choline as a neurotransmitter has important role in the CNS and is implicated in behavioral as well as learning and memory and neurodegenerative diseases. Our results showed that administration of MSG in rats increased AChE activity in the hippocampus. Considering these findings we speculate that increased AChE activity may lead to a reduction of cholinergic neurotransmission efficiency due to a decrease in acetyl choline level in the synaptic cleft, thus contributing to progressive cognitive impairment $[27,28]$. In addition, Abu-Taweel et al. [28] showed that MSG caused oxidative stress by increasing lipid peroxides and reactive oxygen species (ROS), which could enhance AChE activity. Several studies indicate that curcumin possess antioxidant, anti-inflammatory and calcium antagonist 
activities along with memory enhancing effect [29]. In the present study, administration of CUR either alone or concomitant to MSG revealed that AChE activity was marginally recovered towards normal; suggesting its neuroprotective properties.

Consistently, our results show increased levels of TNF- $\alpha$ in hippocampus of MSG group. This confirmed neuro-inflammation by MSG. Activation of microglia in hippocampus results in releasing many neuro-inflammatory mediators such as TNF- $\alpha$, IL-1 $\beta$ and NF- $\kappa B$ [8]. In addition, microglial activation can induce an inflammatory-oxidative cascade leading to cognitive deficit [30]. However, our findings indicate that CUR treatment significantly reduced the levels of this inflammatory factor. These results are similar to the observations of Cho et al. [31] who find a significant reduction of the inflammatory cytokines after treatment with CUR. In addition, other studies reported that CUR can suppress the expression of TNF- $\alpha$ induced by different stimuli [32].

\section{Conclusion}

The ability of overcoming glutamate elevated level by administration of CUR could ameliorate the cognitive impairment of MSG through its maintaining effect on AChE level and reducing TNF $\alpha$ level suggesting that CUR can be used as a protective agent with MSG supplement, and thus we may consider its market application. Curcumin may offer also better neural cell protection, by its decreasing mGLUR5 \& NMDA2B receptor expression which may be, in part; a prospective trial to overcome MSG induced toxicity. It also can be suggested as complementary supplement during MSG intake to ameliorate the flavor associated neurotoxicity and overcome expected other MSG toxicities in other organs.

\section{Acknowledgment}

The authors are thankful to Faculty of Pharmacy, Tanta University (Egypt) as it helped in the funding of the work.

\section{Disclosure Statement}

The author declares no conflict of interest.

\section{References}

1 Veni NK, Karthika D, Devi MS, Rubini MF, Vishalini M, Pradeepa YJ: Analysis of monosodium-glutamate in food products by high-performance thin layer chromatography. J Young Pharm 2010;2:297-300.

2 Ramanathan M, Sivakumar S, Anandvijayakumar PR, Saravanababu C, Rathinavel PP: Neuroprotective evaluation of standardized extract of Centella asiatica in monosodium glutamate treated rats. Indian J Exp Biol 2007;45:425-31.

3 Weil ZM, Norman GJ: The injured nervous system: a Darwinian perspective. Prog Neurobiol 2008;86:4859.

4 Collison, KS, Makhoul NJ, Zaidi, MZ, Al-Rabiah R, Inglis A, Andres BL, Ubungen R, Shoukri M, Al-Mohanna FA: Interactive effects of neonatal exposure to monosodium glutamate and aspartame on glucose homeostasis. Nutr Metab 2012;9:58.

5 Beas-Zárate C, Rivera-Huizar SV, Martinez-Contreras A, Feria-Velasco A, Armendariz-Borunda J: Changes in NMDA- receptor gene expression are associated with neurotoxicity induced neonatally by glutamate in the rat brain. Neurochem Int 2001;39:1-10.

6 Mukherjee S, Manahan-Vaughan D: Role of metabotropic glutamate receptors in persistent forms of hippocampal plasticity and learning. Neuropharmacol 2013;66:65-81.

7 Ma X, Fang F, Song M, Ma S: The effect of isoliquiritigenin on learning and memory impairments induced by high-fat diet via inhibiting TNF- $\alpha$ /JNK/IRS signaling. Biochem Biophys Res Commun 2015;464:1090-1095.

8 Silva B, Sousa L, Miranda A, Vasconcelos A, Reis H, Barcelos L, Arantes R, Teixeira A, Rachid MA: Memory deficit associated with increased brain proinflammatory cytokine levels and neurodegeneration in acute ischemic stroke. Arq Neuropsiquiatr 2015;73:655-659. 
Khalil/Khedr: Modulation of Monosodium Glutamate Neurotoxicity by Curcumin

9 Nampoothiri M, John J, Kumar N, Mudgal J, Kutty G Nampurath, Chamallamudi MR: Modulatory Role of Simvastatin against Aluminium Chloride-Induced Behavioural and Biochemical Changes in Rats. Behav Neurol 2015;2015:210169. doi: 10.1155/2015/210169.

10 Husarova V, Ostatnikova D: Monosodium glutamate toxic effects and their implications for human intake: A review. JMED Research 2013;2013, Article ID 608765, DOI: 10.5171/2013.608765

11 El-Ashmawy N, Khalil R: A review on the role of L-carnitine in the management of tamoxifen side effects in treated women with breast cancer. Tumor Biol 2014;35:2845-2855.

12 Khedr NF, Khedr EG: Antioxidant and anti-inflammatory effects of curcumin on CCl4 - induced liver fibrosis in rats. AJB Sci 2014;6:191-200.

13 Bossù P, Cutuli D, Palladino I, Caporali P, Angelucci F, Laricchiuta D, Gelfo F, De Bartolo P, Caltagirone C, Petrosini L: A single intraperitoneal injection of endotoxin in rats induces long-lasting modifications in behavior and brain protein levels of TNF- $\alpha$ and IL-18. J Neuroinflammation 2012;29:101.

14 Ellman GL, Callaway E: Erythrocyte cholinesterase-levels in mental patients. Nature 1961;23;192:1216.

15 Jiang S, Jing Su J, Yao S, Zhang Y, Cao F, Wang F, Wang H, Jun Li, Shuhua Xi: Fluoride and arsenic exposure impairs learning and memory and decreases mGluR5 expression in the hippocampus and cortex in rats PLoS One 2014;9:e96041.

16 Landau S, Everitt BS: Handbook of statistical analyses using SPSS. USA, Chapman \& Hall/CRC Press LLC, 2004, pp 1-339.

17 Geha R, Beiser A, Ren C, Patterson R, Greenberger P, Grammer LC, Ditto AM, Harris KE, Shaughnessy MA, Yarnold P, Corren J, Saxon A: Multicenter multiphase double blind placebo controlled study to evaluate alleged reactions to monosodium glutamate (MSG). J. Allergy Clin Immunol 1998;101:S243.

18 Meldrum BS: Glutamate as a neurotransmitter in the brain: review of physiology and pathology. J Nutr 2000;130:1007S-15S.

19 Farombi EO, Onyema 00: Monosodium glutamate-induced oxidative damage and genotoxicity in the rat: modulatory role of vitamin C, vitamin E and quercetin. Hum Exp Toxicol 2006;25:251-259.

20 Rubio MD, Drummond JB, Meador-Woodruff JH: Glutamate receptor abnormalities in schizophrenia: implications for innovative treatments. Biomol Ther 2012;20:1-18.

21 Kondratskaya E, Shin MC, Akaike N: Neuronal glutamate transporters regulate synaptic transmission in single synapses on CA1 hippocampal neurons. Brain Res Bull 2010;81:53-60.

22 Shivasharan BD, Nagakannan P, Thippeswamy BS, Veerapur VP: Protective Effect of Calendula officinalis L. flowers against monosodium glutamate induced oxidative stress and excitotoxic brain damage in rats. Indian J Clin Biochem 2013;28:292-298.

23 Zhou Y, Danbolt NC: Glutamate as a neurotransmitter in the healthy brain. J Neural Transm (Vienna) 2014;121:799-817.

24 Hu X, Huang F, Szymusiak M, Liu Y, Wang ZJ: Curcumin attenuates opioid tolerance and dependence by inhibiting Ca2+/calmodulin-dependent protein kinase II $\alpha$ activity. J Pharmacol Exp Ther 2015;352:420428.

25 Lin TY, Lu CW, Huang SK, Wang SJ: Curcumin inhibits glutamate release from rat prefrontal nerve endings by affecting vesicle mobilization. Int J Mol Sci 2012;13:9097-109.

26 Chi, P, Greengard, P, Pyan, TA: Synaptic vesicle mobilization is regulated by distinct synapsin I phosphorylation pathways at different frequencies. Neuron 2003;38:69-78.

27 Jiang H, Zhang XJ: Acetylcholinesterase and apoptosis. A novel perspective for an old enzyme. FEBS J 2008;275:612-617.

28 Abu-Taweel GM, A ZM, Ajarem JS, Ahmad M: Cognitive and biochemical effects of monosodium glutamate and aspartame, administered individually and in combination in male albino mice. Neurotoxicol Teratol 2014;42:60-67.

29 Yadav P, Jadhav SE, Kumar V, Kaul KK, Pant SC, Flora SJ: Protective efficacy of 2-PAMCl, atropine and curcumin against dichlorvos induced toxicity in rats. Interdiscip Toxicol 2012;5:1-8.

30 Noorafshan A, Abdollahifar MA, Karbalay-Doust S, Asadi-Golshan R, Rashidian-Rashidabadi A: Protective effects of curcumin and sertraline on the behavioral changes in chronic variable stress-induced rats. Exp Neurobiol 2013;22:96-106.

31 Cho JW, Lee KS, Kim CW: Curcumin attenuates the expression of IL- 1beta, IL-6, and TNF-alpha as well as cyclin E in TNF-alpha-treated HaCaT cells; NF-kappa B and MAPKs as potential upstream targets. Int J Mol Med 2007;19:469-474.

32 Jain SK, Rains J, Croad J, Larson B, Jones K: Curcumin supplementation lowers TNF-alpha, IL-6, IL-8, and MCP-1 secretion in high glucose-treated cultured monocytes and blood levels of TNF-alpha, IL-6, MCP-1, glucose, and glycosylated hemoglobin in diabetic rats. Antioxid Redox Signal 2009;11:241-249. 